\title{
Igualdad de género en el plan de estudios generales de pregrado en una universidad pública
}

\section{Gender equality in the undergraduate study programs in a public university}

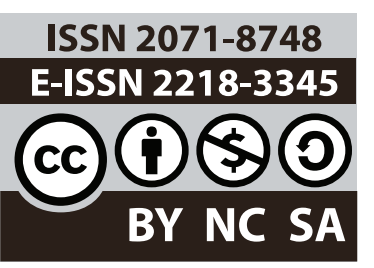

URI: http://hdl.handle.net/11298/896

DOI: http://dx.doi.org/10.5377/entorno.v0i66.6735

\author{
Aymé Barreda-Parra' \\ vbarredapa@unsa.edu.pe \\ ORCID: 0000-0001-7024-0809 \\ Norma Peña-Téllez² \\ npenat@unsa.edu.pe \\ ORCID: 0000-0003-4660-1621 \\ Universidad Nacional de San Agustín \\ Recibido: 29/07/18 \\ Aprobado: 12/12/18
}

\section{Resumen}

El tema de igualdad de género en el plan de estudios generales de pregrado en una universidad pública es pertinente, porque permite a profesores y estudiantes generar espacios de reflexión y promover valores para el ejercicio de sus deberes y derechos. El objetivo fue analizar la inclusión de género en el plan de estudios generales de pregrado para luego reflexionar sobre los resultados. Es un estudio descriptivo transeccional con metodología mixta. Se aplicó una encuesta a 143 estudiantes con edades entre 17 y 22 años, con la finalidad de conocer su opinión en relación a la igualdad de género, después de haber seguido las asignaturas de Razonamiento Lógico Matemático, Ciudadanía e Interculturalidad y Realidad Nacional, las cuales son obligatorias desde el año 2017 de acuerdo a la ley universitaria peruana 30220. Los resultados son alentadores en lo que respecta a la percepción positiva que tienen las mujeres de sus habilidades, pero limitado en la información que poseen sobre su participación a

\section{Abstract}

It is appropriate to include gender equality themes in the common undergraduate study program in a public university for it allows professors and students to create opportunities for reflection and to instill values aimed to exercise rights and duties. The objective was to analyze gender inclusion in the common undergraduate study programs to provide a forum to later reflect on the results.

This being a descriptive trans-sectional study of mixed methodology. A survey was conducted with a group of 143 students aged 17 to 22 years old in order to find out what their opinion was after having completed courses on Logical Mathematical Reasoning, Citizenry and Interculturality, and National Reality, which are mandatory courses as from 2017 in accordance with Peruvian university statutory act 30220 . Results are positive in regards with women's perception of their competencies, however, information is limited when it involves their

1 Departamento de Psicología, Universidad Nacional de San Agustín, Arequipa, Perú

2 Departamento de Psicología, Universidad Nacional de San Agustín, Arequipa, Perú 
cargos públicos. Visualizar el problema permite concluir que en estas asignaturas se trabajan temas de género que permanecen en el recuerdo de los estudiantes; no obstante, persisten los estereotipos que son transmitidos a través de la cultura y que justifican la violencia que ejercen los hombres.

\section{Palabras clave}

Educación superior; Sexismo; Planes de estudio. participation in public affairs. An overview of the issue leads to conclude that gender themes developed in these courses do remain among the students' remembrances, nevertheless, stereotypes persist and these are culturally transmitted thus justifying violence exercised by men.

\section{Keywords}

Higher education; Sexism; Study programs

\section{Introducción}

La población joven en el Perú (de 15 a 29 años de edad) constituye el $27 \%$ de la población total, según datos del Instituto Nacional de Estadística e Informática (INEI, 2015). En esa edad terminan la secundaria y empiezan estudios en la universidad o se insertan al área laboral. Los jóvenes opinan que faltan oportunidades para insertarse al trabajo, pero que la universidad es el espacio educativo que les inspira confianza para el logro de este objetivo, así lo expresaron en la $1^{\text {a }}$ Encuesta Iberoamericana de Juventudes (OIJ, 2013; citado por Espinoza y Choque, 2015).

La universidad también les posibilita, mediante sus escuelas y facultades, generar espacios de práctica cotidiana de los principios y valores asumidos por la comunidad agustina, para que el estudiante sea un ciudadano que conoce, ejerce y defiende sus derechos y cumple sus deberes (Modelo Educativo, 2016). Los derechos y deberes incluyen los orientados a lograr la igualdad de género, ya que el índice general de desigualdad de género en el Perú es de 0.420 (INEI, 2015); y la brecha de género en el mercado laboral es 18 hombres más que mujeres en la actividad económica, lo que significa que de cada 100 hombres en edad de trabajar 82 se encuentran integrando la fuerza laboral (INEI, 2016), es decir, las mujeres tienen mayores obstáculos para insertarse en el mercado laboral una vez terminada su formación en la educación superior.

Instituciones internacionales realizan esfuerzos para disminuir esta brecha. La Organización de las Naciones Unidas (ONU) busca garantizar que todos los estudiantes adquieran conocimientos teóricos y prácticos para promover el desarrollo sostenible, y la Unesco promueve el desarrollo educativo del preescolar a la educación superior en temas de derechos humanos e igualdad de género.

La universidad no es ajena a tales aspiraciones. En la nueva Ley Universitaria peruana "el rechazo a toda forma de violencia, intolerancia y discriminación" es un principio esencial, como es formar profesionales de alta calidad de manera integral y con pleno sentido de responsabilidad social de acuerdo con las necesidades del país. Como consecuencia de las disposiciones contenidas en la nueva ley, se han incorporado en el nuevo plan de estudios generales asignaturas obligatorias y con contenidos dirigidos a la formación integral del estudiante.

Analizar los contenidos de género que están incluidos en el plan de estudios generales es una investigación pionera en la universidad, ya que las asignaturas se desarrollaron por primera vez el año 2017 de acuerdo con la Ley Universitaria n.o 30220 y con la aprobación del Reglamento de Estudios Generales de la Universidad Nacional de San Agustín en el año 2016. Para los nuevos planes curriculares, se han elaborado competencias genéricas, las cuales se desarrollan en un proceso de "aprender haciendo", entendido esto como una actividad en un contexto determinado, que va desde un "saber en acción" a un "saber actuar en contexto" (Juliá, 2015, p. 48).

Enel Perú, las cifras muestran las desigualdades en cuestiones de género, temática abordada en este estudio. Por ejemplo, la violencia contra las mujeres, que es "una de las caras más dolorosas de la discriminación", es un problema social de grandes dimensiones que afecta a mujeres de distintas culturas, condiciones sociales, niveles de educación, edad, etc. (INEI, “Perú: Brechas de Género, 2016”, p. 109). En la 
interrelación de los diferentes actores universitarios existen comportamientos que discriminan y formas de violencia "naturalizadas" que requieren ser reconocidas para tomar acciones; el acoso y el hostigamiento sexual precisan de procedimientos para su prevención, denuncia y eliminación (Fuentes, 2016).

Entonces las razones para analizar la igualdad de género a partir del currículo se basan en a) la existencia de normas internacionales y nacionales, b) la violencia que afecta a más mujeres que hombres, c) las políticas en la educación superior a favor de la igualdad de género y d) los obstáculos para alcanzar la paridad en el área laboral. Por ello, el propósito de la investigación es analizar la inclusión de género en el plan de estudios generales de pregrado y reflexionar sobre los resultados obtenidos a partir de la percepción de los estudiantes.

Se espera que el estudiante adquiera mayor conciencia de la existencia de estas desigualdades, que al culminar sus estudios superiores tenga mejores posibilidades para afrontar esta problemática y que sea capaz de tomar decisiones adecuadas en bien de la sociedad.

\section{Metodología}

Es un estudio descriptivo transeccional con metodología mixta:

Cualitativa. Análisis de contenido de los planes de estudios generales de 1er año que fueron implementados el año 2017.

Cuantitativa. Encuesta a los estudiantes de 2.0 año que el año anterior (2017) se matricularon en las asignaturas de estudios generales.

\section{Participantes}

Se encuestó a 143 estudiantes, 95 mujeres $(66,44 \%)$ y 48 hombres (33,56\%), con edades entre 17 y 22 años de la Escuela Profesional de Psicología.

\section{Instrumento}

Encuesta. Elaborada por las investigadoras con base en el contenido de temas de género en las asignaturas de estudios generales de pregrado.
La encuesta consta de dos partes:

1. Datos generales: sexo, edad, año de estudios.

2. Preguntas para conocer si los estudiantes recuerdan que los docentes trataron temas de género en su asignatura y preguntas para conocer la opinión que tienen los estudiantes de la igualdad de género después de su experiencia en la asignatura.

\section{Procedimiento}

Finalizado el año académico 2017, se procedió a la revisión y al análisis de los planes de estudios generales de la universidad, luego se seleccionaron los sílabos de las asignaturas que incluían temas de género para revisarlos en físico.

A partir de la revisión de los temas de género incluidos en los sílabos, se elaboró la encuesta para cada asignatura y fue aplicada en el mes de abril de 2018 a los estudiantes de 2.0 año que el año previo llevaron por primera vez las asignaturas de estudios generales.

\section{Resultados y discusión}

En la tabla 1 se muestran los resultados del análisis de los sílabos, y en los gráficos, el resultado de las encuestas.

\section{Análisis de los sílabos}

La universidad clasifica el plan de estudios generales en:

Asignaturas generales: Capacidades de aprendizaje

- Comunicación Integral

- Metodología del Trabajo Intelectual Universitario

- Razonamiento Lógico Matemático

Asignaturas generales: Formación Humanística

- Ciudadanía e interculturalidad

- Ecología y Medio Ambiente

- Realidad Nacional

- Ética General y Profesional (se implementará en años superiores, por lo que no ha sido considerada) 
Tabla 1. Análisis de las asignaturas de estudios generales

\begin{tabular}{lll}
\hline Asignatura & $\begin{array}{l}\text { Existen } \\
\text { contenidos } \\
\text { relacionados } \\
\text { con género }\end{array}$ & Contenidos relacionados con género \\
\hline Comunicación Integral & No & \\
\hline $\begin{array}{l}\text { Metodología del Trabajo } \\
\text { Intelectual Universitario }\end{array}$ & No & \\
\hline Ecología y Medio Ambiente & No & Confianza en las habilidades para plantear y resolver \\
Razonamiento Lógico Matemático & Sí & problemas (como parte del contenido actitudinal) \\
\hline Ciudadanía e Interculturalidad & Sí & Participación ciudadana \\
\hline Realidad Nacional & Sí & Violencia y poder político \\
\hline
\end{tabular}

Los contenidos relacionados con género fueron agrupados en 3 categorías de acuerdo con la similitud de su significado: género, igualdad-equidad y política del género (Rebollar, 2013). A continuación, los contenidos incluidos en cada categoría:
Género: estereotipos (habilidades para las matemáticas) Igualdad-equidad: discriminación (violencia)

Política del género: participación ciudadana y poder político

\section{Asignatura Razonamiento Lógico Matemático}

\section{Gráfico 1. Percepción de confianza en las habilidades para las matemáticas}

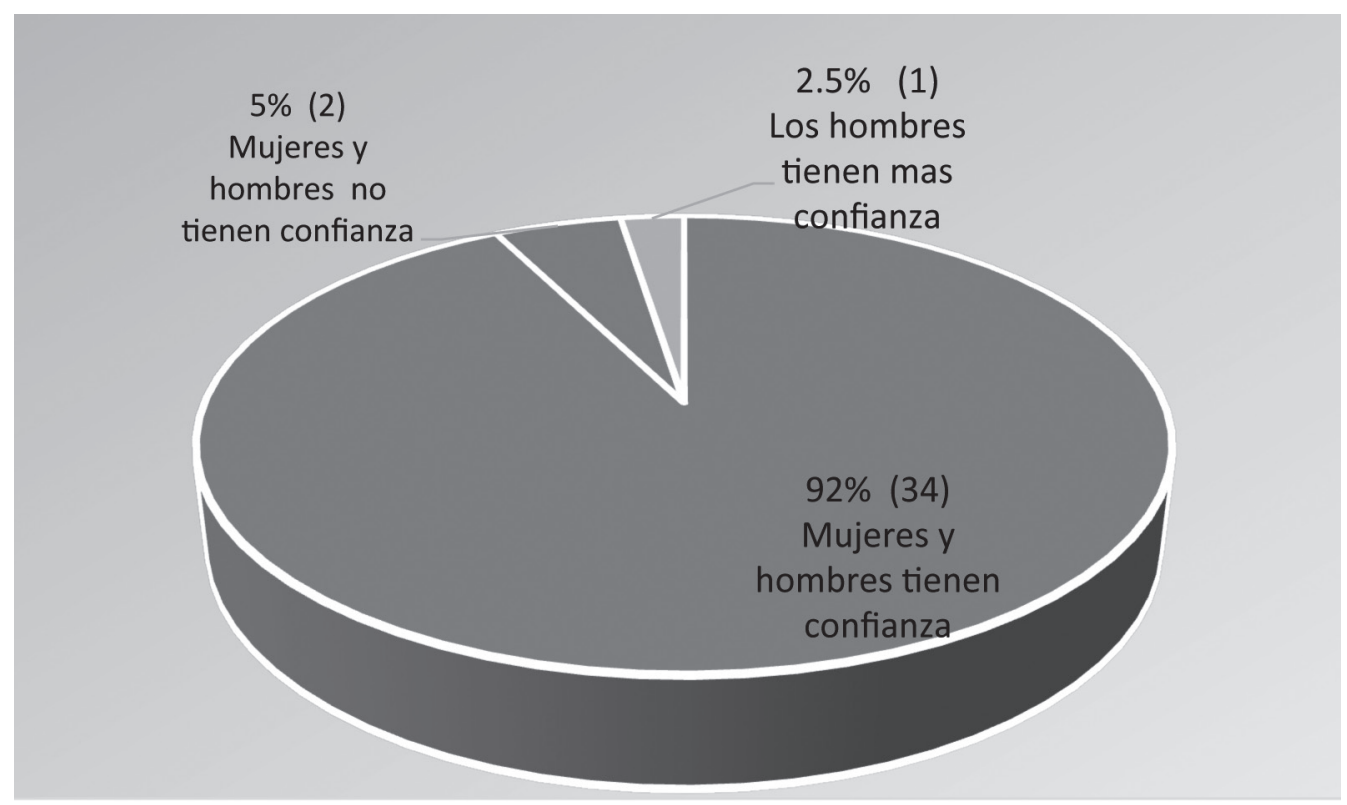


Asignatura Ciudadanía e Interculturalidad

Gráfico 2. Percepción de las mujeres en igualdad-equidad y política de género

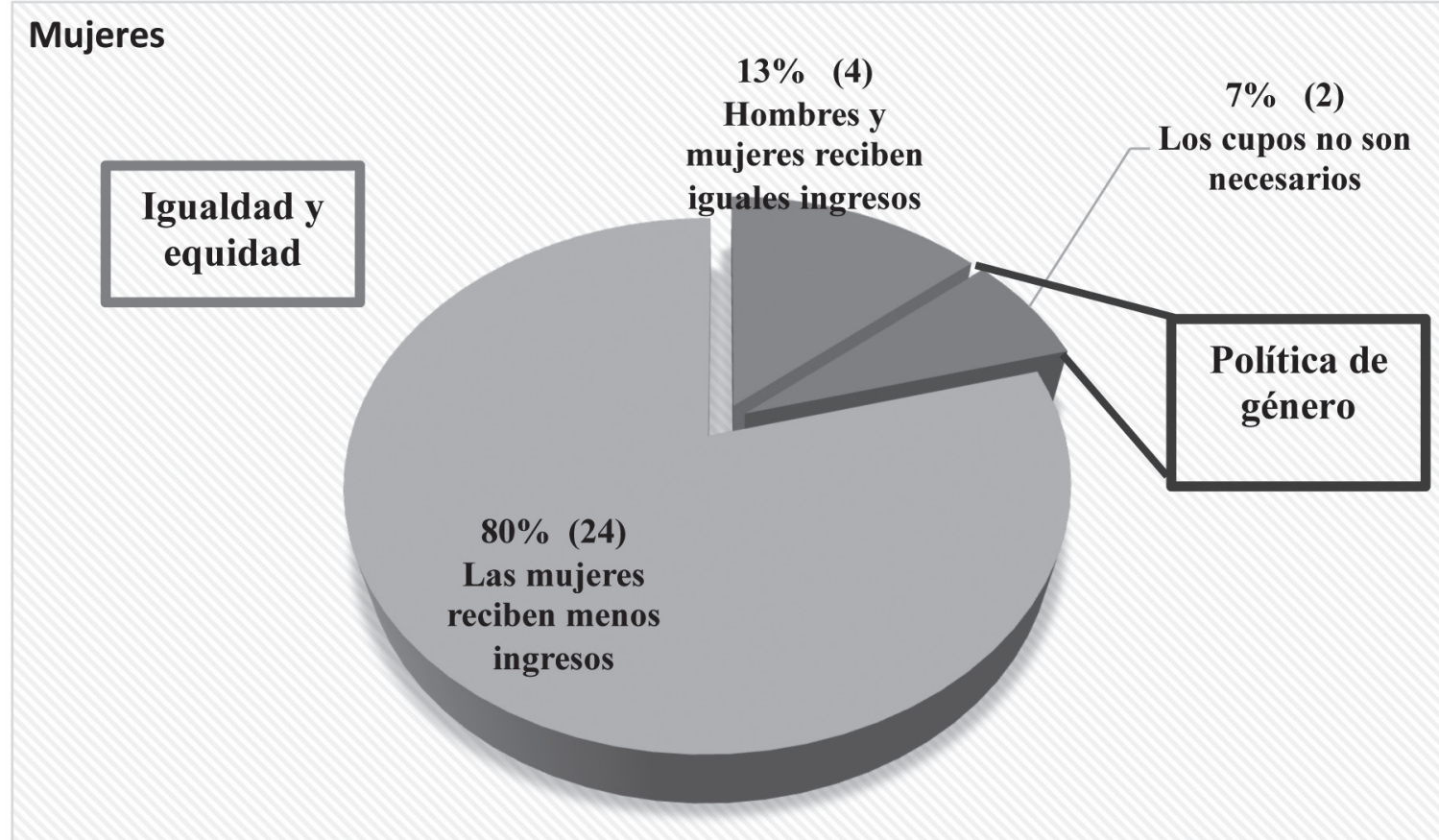

Gráfico 3. Percepción de los hombres en igualdad-equidad y política de género

\section{Hombres}

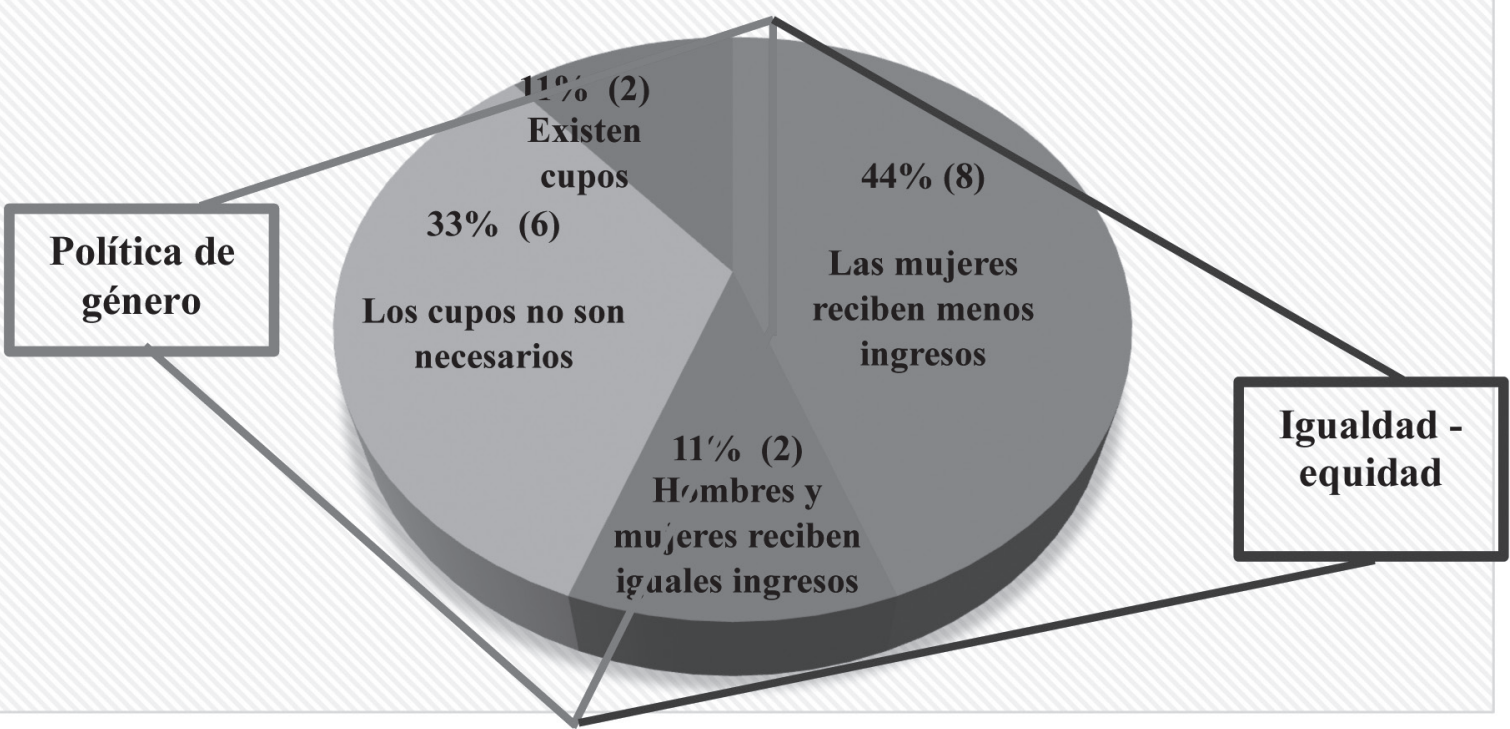




\section{Asignatura de Realidad Nacional}

\section{Gráfico 4. Percepción de los hombres en igualdad-equidad y política de género por sexo}

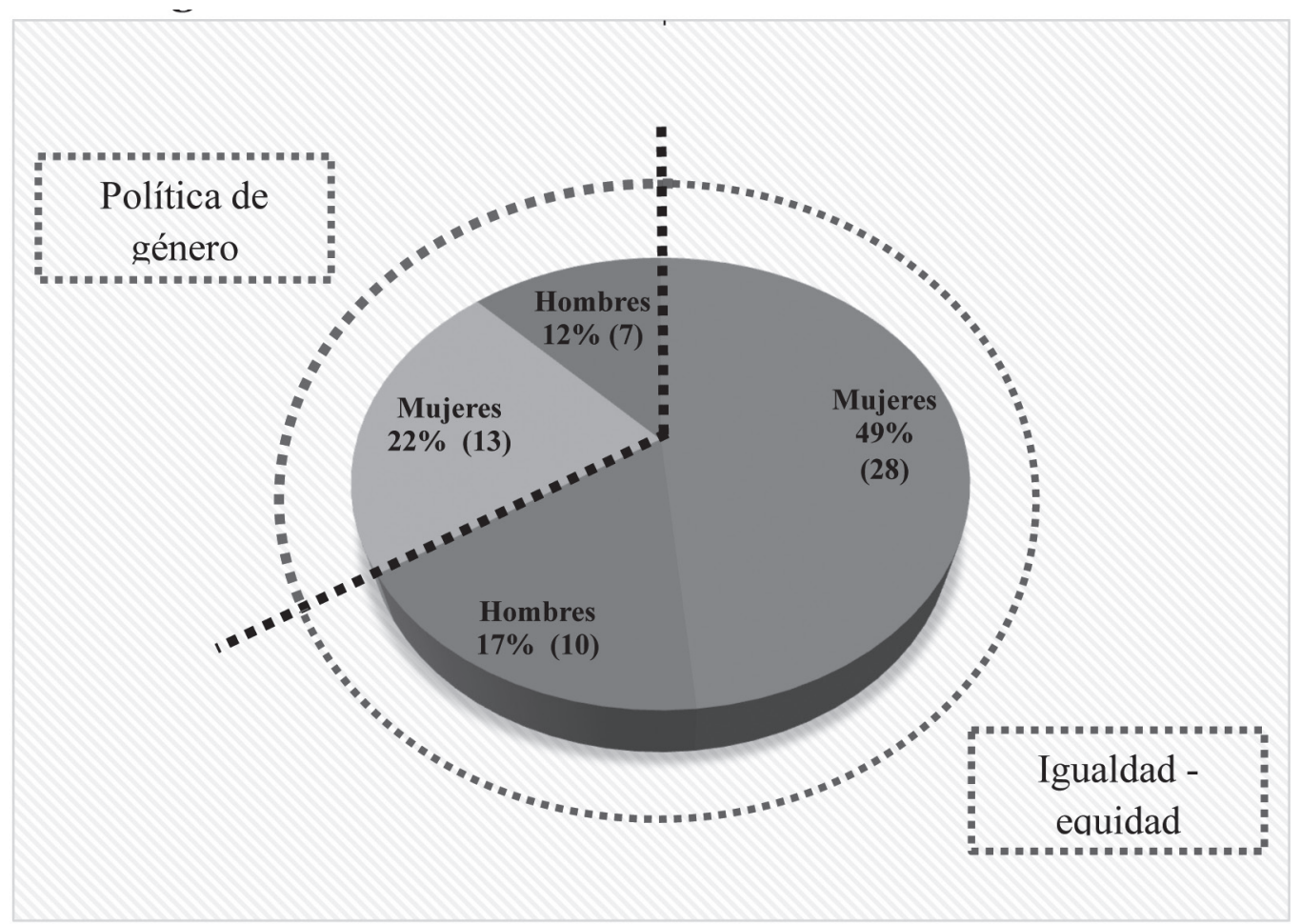

En la universidad, la inclusión de los estudios generales ha implicado un cambio significativo en la formación del estudiante, son obligatorios en todas las carreras profesionales; y con la perspectiva de que el estudiante asuma compromiso consigo mismo y con la sociedad. Por otro lado, es un gran avance que en el currículo estén incluidos temas de género. No obstante, se requiere profundizar en el aula estos contenidos, para que los estudiantes tomen conciencia de las desigualdades de género en diversos ámbitos donde se desempeña la mujer. La universidad es un lugar propicio para establecer relaciones interpersonales entre los jóvenes; sin embargo, la continua interacción entre las personas genera relaciones de poder y subordinación. El poder ocupa un papel importante en las interacciones debido a las diferentes variables que intervienen en su configuración, como la propia personalidad de la joven y el joven, sus estereotipos, prejuicios, status social, descalificación de la mujer o esquemas que traen consigo los papeles que se les asignan a mujeres y hombres.
Las asignaturas analizadas son las siguientes: Razonamiento Lógico Matemático, Ciudadanía e Interculturalidad y Realidad Nacional.

El resultado (92\%) obtenido en esta asignatura por mujeres y hombres es esperanzador y positivo. Las mujeres opinan que sus habilidades para las matemáticas son tan favorables como las de los hombres. Esto es un indicador de que la brecha de género en ciencias e ingenierías está cambiando en el tiempo. A esta conclusión llegaron Sax et al. (2016) después de examinar los factores determinantes de los universitarios de primer año para especializarse en ingeniería. El estudio reveló que un factor determinante en el interés por la especialización es el rendimiento académico obtenido en la secundaria, para hombres y mujeres; sin embargo, el autoconcepto matemático que en el pasado fue un factor importante en la brecha de género ahora es menos influyente en la intención de las mujeres para estudiar ciencias e ingenierías. Las mujeres que estudian 
Psicología no eligieron estudiar Ingeniería, pero es un buen indicador de que perciben confianza en sus habilidades para las matemáticas; porque durante la carrera llevarán otros cursos afines, como estadística.

Ciudadanía e Interculturalidad. Participación ciudadana contempla los espacios donde los ciudadanos ejercen su derecho a votar y ser elegidos en cargos públicos; se relaciona con la democracia participativa y con la libertad de expresión. Algunos estudiantes, mujeres (13\%) y hombres (11\%), opinaron que ambos sexos reciben iguales ingresos económicos en el mismo cargo; a pesar de no ser tan reales estas cifras. Por ejemplo, el año 2015 las mujeres ganaron en promedio $28,6 \%$ menos que sus pares masculinos. Uno de los motivos principales por el que las mujeres ganan menos es que muchas de ellas trabajan menos horas, por dedicarse a sus familias (INEI Brechas de Género 2016).

En relación con los cupos de participación para las elecciones, más hombres (33 \%) que mujeres (7\%) opinan que no son necesarios. Existen leyes creadas para fomentar la participación de las mujeres en cargos directivos de decisión política, pero todavía no se ha logrado la paridad con los hombres. La cuota mínima de participación de mujeres y hombres en las listas de candidatos al Congreso de la República es 30 \% (Ley n.o 27387), igualmente $30 \%$ en las listas de candidatos a los Consejos Regionales (Ley n.o 27683) y candidatos a regidores [Ley n.o 26864] (Espinoza, 2009).

Realidad Nacional. Respecto a la violencia, más mujeres (49\%) que hombres (17\%) consideran que los hombres tienen poco control de sus emociones y ejercen violencia en contra de las mujeres. Respuesta similar a la obtenida por Mingo (2016); y que es una forma de disfrazar la verdad acerca de la sexualidad "supuestamente irreprimible" de los hombres con el mensaje tácito de que toca a las mujeres cuidar que el deseo sexual de los hombres no se despierte. Es un mito "pensar que todos los hombres nacen para ser violentos" (Castro, 2015). La realidad demuestra que hay vinculación entre violencia y cultura. Coexisten variables socioculturales de la familia, la escuela y los medios de comunicación que distorsionan el significado de la masculinidad, otorgándole al hombre un poder que mal utilizado lo conduce a comportamientos discriminatorios y violentos.

Los estudiantes, en la asignatura Realidad Nacional percibieron como más importante la violencia, y en menor proporción (22\% de mujeres y $7 \%$ de hombres) la alternativa de que las mujeres hacen buena gestión cuando ocupan un cargo público. Puede haber influido la exposición de noticias de violencia, abuso sexual y feminicidios que diariamente trasmiten los medios de comunicación. Es necesario que los estudiantes obtengan mayor información de la gestión que hacen las mujeres en cargos directivos donde ejercen la toma de decisiones. Como gerentes públicos, en el Perú muestran que entre el 2012 y el 2015 las mujeres tienen tan igual desempeño como los hombres. Así lo demuestra la evaluación de desempeño donde $97 \%$ superó el $70 \%$ de sus metas. El año 2012 fue el de menor desempeño (86 \% y $84 \%$, en promedio, para las mujeres y los hombres, respectivamente). No obstante, el porcentaje de desempeño ha estado por encima del $97 \%$ en los años 2013, 2014 y 2015, tanto para los hombres como para las mujeres (SERVIR, 2016).

En conclusión, es una experiencia positiva que de 6 asignaturas de estudios generales en 3 se incluyan contenidos de género en el sílabo. Los jóvenes estudiantes de 2.0 año recuerdan los temas de género tratados en estas últimas, si bien, como demuestran los resultados, hay elementos esenciales de la brecha de género en los cuales se debe profundizar, tales como las cuotas de participación que son necesarias mientras las mujeres no alcancen la tan ansiada paridad en la toma de decisiones y el mejor entendimiento de las causas de la violencia; pero es evidente que los estudiantes de ambos sexos perciben que tienen habilidades para las matemáticas.

En próximas investigaciones, sería conveniente contrastar la percepción que tienen los estudiantes con sus notas en la asignatura Razonamiento Lógico Matemático. Por otro lado, consideramos una limitación no haber entrevistado a los profesores que tuvieron a su cargo las asignaturas para dar mayor validez a los datos obtenidos.

Las asignaturas de estudios generales de la universidad amplían la formación del estudiante más allá de los cursos de especialidad de cada carrera, con el objetivo de promover el desarrollo de la persona humana. Para mejorar la cobertura y los procedimientos en cuestiones de género sería conveniente la creación de una Unidad de Igualdad similar a las creadas en otras universidades (Ley Orgánica 4/2007, España). Finalmente, un ambiente universitario saludable fomenta los valores éticos, para una convivencia sin violencia y con igualdad de derechos para mujeres y hombres, por lo que se recomienda más información, 
diálogo y debate en clase para aclarar las dudas que tienen los estudiantes.

\section{Referencias}

Castro, R. (2015). "Desmontando la Violencia". Recuperado de http://www.conigualdad.org/descargas/ Desmontando\%20la\%20violencia\%20de\%20Genero_ RubenCastro.pdf

Espinoza, E. (2009). “Cuotas de participación de las mujeres en cargos directivos de decisión política". Documento preliminar. Lima-Perú. Recuperado de https://www. mimp.gob.pe/files/direcciones/dgignd/documentos/ informe_cuotas_genero.pdf

Espinoza-Lecca, E. y Choque-Larrauri, R. (2015). “El Estado de la Juventud en el Perú: Situación actual e iniciativa para un cambio". En: Evidencia para políticas públicas en educación superior, Vol. 1. Hans Contreras Pulache Editor. Programa Nacional de Becas y Crédito Educativo. Ministerio de Educación. Recuperado de https://www.inei.gob.pe/prensa/noticias/el-27-de-lapoblacion-peruana-son-jovenes-8547/

Fuentes, L.Y. (2016). “¿Por qué se requieren políticas de equidad de género en la educación superior?". Nómadas, 44, 65-83. Recuperado de http://www. scielo.org.co/pdf/noma/n44/n44a05.pdf

INEI (2015). "El 27 \% de la población peruana son jóvenes". Notas de prensa. Recuperado el 02 de julio de 2018 de https://www.inei.gob.pe/prensa/noticias/el-27-de-lapoblacion-peruana-son-jovenes-8547/

INEI (2016). “Perú: Brechas de género 2016. Avances hacia la igualdad de mujeres y hombres". pp. 13-14. Recuperado el 12 de diciembre de 2018 de http://www.inei.gob.pe/ media/MenuRecursivo/publicaciones_digitales/Est/ Lib1388/Libro.pdf

Juliá, M. (2015). “Competencias generales de la formación universitaria: Aportes a la calidad con equidad". En G. Carrillo (Ed.), I Encuentro Internacional Universitario. El currículo por competencias en la Educación Superior. Ponencias y debate. Vicerrectorado Académico. Dirección de Asuntos Académicos PUCP.

Ley Orgánica 4/2007, de 12 de abril, por la que se modifica la Ley Orgánica 6/2001, de 21 de diciembre, de Universidades. Disposición adicional duodécima.
Unidades de igualdad. España. Recuperado de https:// www.boe.es/boe/dias/2007/04/13/pdfs/A1624116260.pdf

Mingo. A. (2016). "'¡Pasen a borrar el pizarrón!'. Mujeres en la universidad". Revista de la Educación Superior. 45, (178), 1-15. Recuperado el 12 de diciembre de 2017 de https://www.sciencedirect.com/science/article/pii/ S0185276016300036?via\%3Dihub

Modelo Educativo de la Universidad Nacional de San Agustín (2016) Resolución de Consejo universitario n.o 513-2016 Nueva Ley Universitaria n.o 30220 - 2014. Artículo 5, Inciso 5.16. La Nueva Ley Universitaria n.o 30220 - 2014. Diario Oficial El Peruano. Normas Legales. Año XXXI n.o 12914. 9 de julio de 2014. Recuperado de https:// www.sunedu.gob.pe/wp-content/uploads/2017/04/ Ley-universitaria-30220.pdf

ONU. "ODS en la Agenda 2030 de las Naciones Unidas: Retos de los Objetivos de Desarrollo Sostenible". Observatorio de la Cooperación Universitaria al Desarrollo. Recuperado de http://www.ocud.es/agenda2030/es/ thm4/objetivos-de-desarrollo-sostenible.htm

Rebollar, E. (2013). "El Género en los planes de estudio de los grados de educación en las universidades públicas españolas" (tesis doctoral). Universidad Autónoma de Barcelona, España. Recuperado de https://ddd.uab. cat/record/118631

"Reglamento deestudiosgenerales dela Universidad Nacional de San Agustín". (2016). Recuperado de http://www. unsa.edu.pe/transparencia/docs/REGLAMENTO $\% 20$ DE\%20ESTUDIOS\%20GENERALES\%20DE\%20LA\%20 UNSA.pdf

Sax, L.J.; Kanny, M.A.; Jacobs, J.A.; Whang, H.; Weintraub, D.S., \& Hroch, A. (2016). "Understanding the Changing Dynamics of the Gender Gap in Undergraduate Engineering Majors: 1971-2011". Research In Higher Education, 57, 570. Recuperado de https://doi. org/10.1007/s11162-015-9396-5

SERVIR. Autoridad Nacional del Servicio Civil. Perú. (2016). Perú: Presidencia del Consejo de Ministros. Recuperado de http://www.servir.gob.pe/nosotros/ consejo-directivo/

Unesco. “Educación para el Siglo XXI. La educación transforma vidas. La labor de la Unesco". Recuperado de https:// es.unesco.org/themes/education-21st-century 